\title{
HAMILTONIAN FORMULATION OF PLANAR BEAMS
}

\author{
Goran Golo ${ }^{*, * * 1}$ Arjan van der Schaft ${ }^{*, 1}$ \\ Stefano Stramigioli ${ }^{* * *, 1}$ \\ * Department of Appl. Mathematics, University of Twente \\ P.O. Box 217, 7500 AE Enschede, The Netherlands \\ ** ControlLab Products B.V., The Netherlands \\ *** Department of Elect. Engineering, University of Twente \\ P.O. Box 217, 7500 AE Enschede, The Netherlands
}

\begin{abstract}
This paper deals with the port-Hamiltonian formulation of planar beams. It is shown how this formulation can be related to the linear beams models: the Timoshenko beam model and the rod model. Copyright (C) 2003 IFAC
\end{abstract}

Keywords: Port-Hamiltonian systems, Dirac structures, planar beams

\section{INTRODUCTION}

In previous work, see e.g. (van der Schaft and Maschke, 1995), (Dalsmo and van der Schaft, 1999), (van der Schaft, 2000), it has been shown how port-based network modeling of lumpedparameter physical systems naturally leads to a generalized Hamiltonian formulation of the dynamics. In fact, the Hamiltonian is given by the total energy of the energy-storing elements in the system, while the geometric structure, defining together with this Hamiltonian the dynamics of the system, is given by the power-conserving interconnection structure of the system (corresponding to what in bond-graph terminology is called the "generalized junction structure"), and is called a Dirac structure. Furthermore, there energydissipating elements may be added by terminating some of the system ports. The resulting class of open dynamical systems has been called "portHamiltonian systems" (van der Schaft, 2000).

1 The work was supported by the EU-project GeoPlex EUIST-2002-34166
The identification of the Hamiltonian structure of the dynamical model is important for various reasons. From a simulation point of view it immediately yields information about the energy and other conserved quantities in the system, which preferably should be respected in simulation routines. Furthermore, it is instrumental in finding the most convenient representation of the equations of motion of the system; in the format of purely differential equations or of mixed sets of differential and algebraic equations (DAEs), see e.g (Golo, 2002). From an analysis point of view it allows to use the powerful methods regarding stability from the theory of dynamical Hamiltonian systems. Finally, the Hamiltonian structure may fruitfully be used in the control design, e.g. by the explicit use of the energy function and conserved quantities (perhaps after feedback) for the construction of a Lyapunov function. We refer to (Ortega et al., 2001), (van der Schaft, 2000) for various work in this direction.

Recently, the framework of port-Hamiltonian systems has been extended to classes of distributedparameter systems (van der Schaft and Maschke, 
2002), like Maxwell's equations over a bounded domain with energy radiation through its boundary, the $n$-dimensional wave equation, and compressible ideal fluids. Hereto a special type of infinite-dimensional Dirac structure has been introduced, based on Stokes theorem. Physically, this Stokes-Dirac structure captures the basic balance laws of the system, like Faradays and Ampères law, or mass balance and can be seen as a geometric continuous distributed form of Tellegen's theorem. The port-Hamiltonian formulation is a non-trivial extension of the Hamiltonian formulation of PDEs by means of Poisson structures, as has been explored before in the literature (Olver, 1993). Indeed, in this case it is crucially assumed that the boundary conditions are such that the energy-flow through the boundary of the spatial domain is zero. In order to allow a non-zero boundary energy-flow the use of Dirac structures instead of Poisson structures seems to be indispensable. As a result complex physical systems consisting of components which are either lumpedparameter or distributed-parameter systems, and which moreover may belong to different physical domains (mechanical, electrical, hydraulic, ..), can be modeled in a unified and intrinsic way. As before, this opens up several possibilities for the analysis and control of such complex systems, making use of the Hamiltonian structure in an explicit way.

The goal of this paper is to develop a dynamical model of planar beams which encompasses large deformations. For a nice geometric description of the statics of a beam using screw theory the reader can consult (Selig and Ding, 2001). A finite element of the beam is considered as a rigid body connected to other elements via flexible joints. The corresponding continuous model represents a Port-Hamiltonian system with modulated interconnection structure (Dirac structure is nonlinear). If the non-linear terms are neglected then the simplified model can be split into two parts. The first part describes longitudinal motion of the beam (rod model) and the second part represents transversal motion of beam (Timoshenko beam model).

\section{BEAM MODEL}

Consider a beam shown in Figure 1. The spatial variable $s \in[0, S]$ defines a position along the unstressed beam with length $S$. A position of a point of the beam is defined by $(x(s), y(s))$ and the angle $\theta(s)$ stands for the the rotation of the neutral axis with respect to a horizontal reference. The beam is uniformly partitioned as shown in Figure 2. Every element represents a rigid body connected to other two adjoining elements via

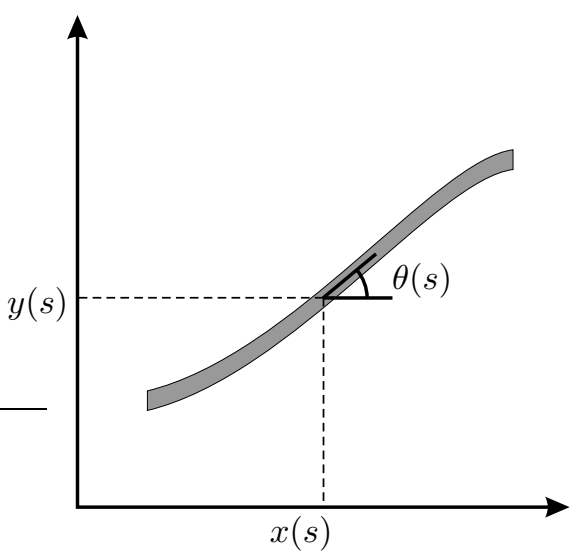

Fig. 1. Stressed beam.

flexible joints (see Figure 2). The length of an element is denoted by $a$.

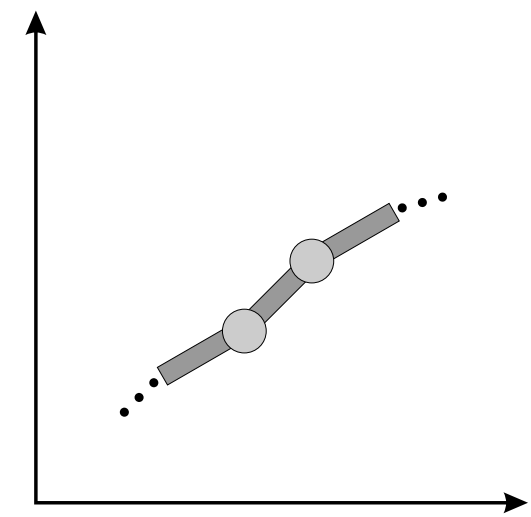

Fig. 2. Partitioned beam.

A finite element of the beam is shown in Figure 3 . The velocity of the center of mass is denoted by $v$, and $\omega$ stands for the angular velocity of the element. The sum of the longitudinal and shear forces is denoted by $F$, and $M$ stands for the bending moment. The inertial frame is denoted by $\Psi_{N}$ and the body frame of the considered part is denoted by $\Psi(s)$. Frames attached to the joints are denoted by $\Psi_{J}(s)$ and $\Psi_{J}(s+a)$ (see figure $3)$.

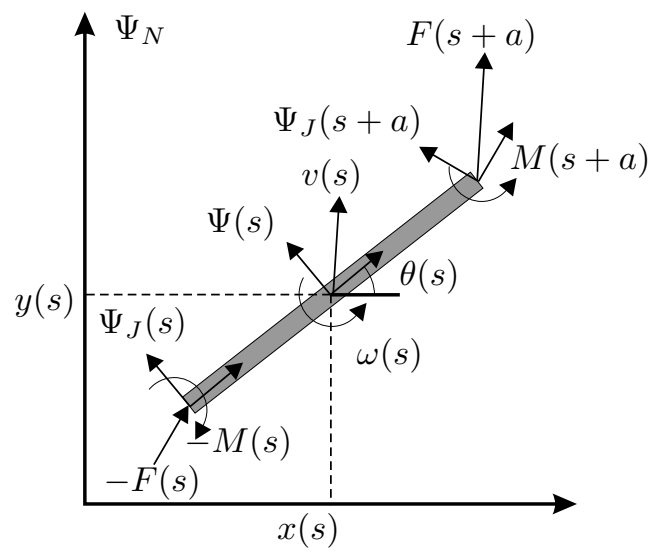

Fig. 3. Finite element of the beam. 


\subsection{Interconnection structures}

In this subsection we derive equations describing the interconnection structure of the beam. Let $t_{b}(s)$ stands for the twist representing the motion of the body frame $\Psi(s)$ with respect to the inertial frame $\Psi_{N}$ and expressed in the body frame $\Psi(s)$. The components of $t_{b}(s)$ are

$$
t_{b}(s)=\left[\begin{array}{c}
\omega(s) \\
v_{x}(s) \\
v_{y}(s)
\end{array}\right]
$$

Here, $v_{x}$ is the longitudinal velocity and $v_{y}$ is the transversal velocity. The motion between the body and the inertial frame expressed in the inertial frame is denoted by $t_{n}(s)$. The twists $t_{b}(s), t_{n}(s)$ are related as (see (Stramigioli, 2001) for details)

$$
t_{n}(s)=\operatorname{Ad}_{b}^{n}(s) t_{b}(s),
$$

where

$$
\operatorname{Ad}_{b}^{n}(s)=\left[\begin{array}{ccc}
1 & 0 & 0 \\
y(s) & \cos (\theta(s)) & -\sin (\theta(s)) \\
-x(s) & \sin (\theta(s)) & \cos (\theta(s))
\end{array}\right] .
$$

Consequently,

$$
t_{n}(s-a)=\operatorname{Ad}_{b}^{n}(s-a) t_{b}(s-a),
$$

Furthermore, the relative motion between two connected rigid bodies expressed in the inertial frame is

$$
t_{n}(s)-t_{n}(s-a) \text {. }
$$

This motion expressed in the frame $\Psi_{J}(s)$ is denoted by $t_{\delta}(s)$ and given by

$$
t_{\delta}(s)=\operatorname{Ad}_{b}^{J} \operatorname{Ad}_{n}^{b}(s)\left(t_{n}(s)-t_{n}(s-a)\right),
$$

where

$$
\operatorname{Ad}_{n}^{b}(s)=\left(\operatorname{Ad}_{b}^{n}(s)^{-1}\right.
$$

and

$$
\operatorname{Ad}_{b}^{J}=\left[\begin{array}{ccc}
1 & 0 & 0 \\
0 & 1 & 0 \\
\frac{1}{2} a & 0 & 1
\end{array}\right] .
$$

Therefore, the relative motion $t_{\delta}(s)$ has the following form

$t_{\delta}(s)=\operatorname{Ad}_{b}^{J} \operatorname{Ad}_{n}^{b}(s)\left(\operatorname{Ad}_{b}^{n}(s) t_{b}(s)-\operatorname{Ad}_{b}^{n}(s-a) t_{b}(s-a)\right)$.

If $a$ tends to zero then the previous expression becomes $\left(\operatorname{Ad}_{b}^{J} \rightarrow I_{3}\right)$

$$
t_{\delta}=\operatorname{Ad}_{n}^{b} \mathrm{~d}\left(\operatorname{Ad}_{b}^{n} t_{b}\right)
$$

Here the operator d represents the exterior derivative. Let $w_{\delta}(s)$ represents the wrench generated by elastic forces and expressed in the frame $\Psi_{J}(s)$. The components of the wrench $w_{\delta}(s)$ are

$$
w_{\delta}(s)=\left[\begin{array}{c}
M(s) \\
F_{x}(s) \\
F_{y}(s)
\end{array}\right] \text {. }
$$

Here, $F_{x}$ stands for the longitudinal forces, $F_{y}$ for the shear forces and $M$ for the bending torque. Let $w_{n}(s)$ stands for the wrench generated by elastic forces and expressed in the frame $\Psi_{N}$. The wrench $w_{n}(s)$ is related to $w_{\delta}(s)$ as (see (Stramigioli, 2001) for details)

$$
w_{n}(s)=\left(\operatorname{Ad}_{n}^{b}(s)\right)^{\mathrm{T}}\left(\operatorname{Ad}_{b}^{J}\right)^{\mathrm{T}} w_{\delta}(s) .
$$

Consequently,

$$
w_{n}(s+a)=\left(\operatorname{Ad}_{n}^{b}(s+a)\right)^{\mathrm{T}}\left(\operatorname{Ad}_{b}^{J}\right)^{\mathrm{T}} w_{\delta}(s+a) .
$$

Therefore, the total wrench applied to the rigid body is then

$$
w_{n}(s+a)-w_{n}(s) .
$$

This wrench expressed in the reference frame $\Psi(s)$ is denoted by $w_{b}(s)$ and has the following form

$$
\begin{gathered}
w_{b}(s)=\left(\operatorname{Ad}_{b}^{n}(s)\right)^{\mathrm{T}}\left(w_{n}(s+a)-w_{n}(s)\right)= \\
\left(\operatorname{Ad}_{b}^{n}(s)\right)^{\mathrm{T}}\left(\operatorname{Ad}_{n}^{b}(s+a)\right)^{\mathrm{T}}\left(\operatorname{Ad}_{b}^{J}\right)^{\mathrm{T}} w_{\delta}(s+a) \\
-\left(\operatorname{Ad}_{b}^{n}(s)\right)^{\mathrm{T}}\left(\operatorname{Ad}_{n}^{b}(s)\right)^{\mathrm{T}}\left(\operatorname{Ad}_{b}^{J}\right)^{\mathrm{T}} w_{\delta}(s) .
\end{gathered}
$$

If $a$ tends to zero then the previous expression becomes

$$
w_{b}=\left(\operatorname{Ad}_{b}^{n}\right)^{\mathrm{T}} \mathrm{d}\left(\left(\operatorname{Ad}_{n}^{b}\right)^{\mathrm{T}} w_{\delta}\right) .
$$

The boundary variables are denoted by $t_{b}^{0}, w_{b}^{0}$, $t_{b}^{S}, w_{b}^{S}$. The left boundary variables are the twist $t_{b}^{0}$ and wrench $w_{b}^{0}$ expressed in the frame $\Psi(0)$. Similarly, the right boundary variables are the twist $t_{b}^{S}$ and the wrench $w_{b}^{S}$ expressed in the frame $\Psi(S)$. The relations between the spatial variables $t_{b}(s), t_{w}(s)$ and the boundary variables are

$$
\begin{gathered}
t_{b}(0)=t_{b}^{0}, w_{b}(0)=w_{b}^{0} \\
t_{b}(S)=t_{b}^{S}, w_{b}(S)=w_{b}^{S} .
\end{gathered}
$$

In the sequel we prove that the space of admissible twists and wrenches described by Equations (1), $(2),(3)$ is a Dirac structure. Let the space of flows be defined as

$$
\mathcal{F}=\Omega_{1}^{3}[0, S] \times \Omega_{0}^{3}[0, S] \times \mathbb{R}^{3} \times \mathbb{R}^{3} .
$$

Here $\Omega_{0}[0, S]$ stands for the space of zero-forms and $\Omega_{1}[0, S]$ for the space of one-forms. An element of the space $\mathcal{F}$ is denoted by $f$ and represented by

$$
f=\left[\begin{array}{c}
t_{\delta} \\
t_{b} \\
t_{b}^{0} \\
t_{b}^{S}
\end{array}\right] .
$$

Clearly, $t_{\delta}$ is the vector whose components are one-forms and $t_{b}$ is the vector whose components are zero-forms. Let the space of efforts be defined as

$$
\mathcal{E}=\Omega_{0}^{3}[0, S] \times \Omega_{1}^{3}[0, S] \times \mathbb{R}^{3} \times \mathbb{R}^{3} .
$$

An element of the space $\mathcal{E}$ is denoted by $e$ and represented by

$$
e=\left[\begin{array}{l}
w_{\delta} \\
w_{b} \\
w_{b}^{0} \\
w_{b}^{S}
\end{array}\right] .
$$

The power product $\langle\cdot \mid \cdot\rangle: \mathcal{F} \times \mathcal{F} \rightarrow \mathbb{R}$ is defined by 


$$
\begin{aligned}
\langle f \mid e\rangle:= & \int_{0}^{S}\left(w_{\delta}(s)\right)^{\mathrm{T}} t_{\delta}(s)+\int_{0}^{S}\left(w_{b}(s)\right)^{\mathrm{T}} t_{b}(s)+ \\
& \left(w_{b}^{0}\right)^{\mathrm{T}} t_{b}^{0}-\left(w_{b}^{S}\right)^{\mathrm{T}} t_{b}^{S} .
\end{aligned}
$$

The space of admissible flows and effort imposed by Equations (1), (2), (3) is denoted by $\mathcal{D}$ and given by

$$
\begin{aligned}
\mathcal{D}= & \left\{(f, e) \in \mathcal{F} \times \mathcal{E}: t_{\delta}=P t_{b}, w_{b}=P^{*} w_{\delta}\right. \\
& t_{b}(0)=t_{b}^{0}, w_{b}(0)=w_{b}^{0}, \\
& \left.t_{b}(S)=t_{b}^{S}, w_{b}(S)=w_{b}^{S}\right\} .
\end{aligned}
$$

Here the linear transformations $P: \Omega_{0}^{3}[0, S] \rightarrow$ $\Omega_{1}^{3}[0, S], P^{*}: \Omega_{0}^{3}[0, S] \rightarrow \Omega_{1}^{3}[0, S]$ are given by

$$
\begin{aligned}
P & =\operatorname{Ad}_{n}^{b} \mathrm{dAd}_{b}^{n}, \\
P^{*} & =\left(\operatorname{Ad}_{b}^{n}\right)^{\mathrm{T}} \mathrm{d}\left(\operatorname{Ad}_{n}^{b}\right)^{\mathrm{T}} .
\end{aligned}
$$

Theorem 1. The subspace $\mathcal{D} \subset \mathcal{F} \times \mathcal{E}$ is a Dirac structure with respect to the bilinear form

$$
\ll\left(f^{1}, e^{1}\right),\left(f^{2}, e^{2}\right) \gg=\left\langle e^{1} \mid f^{2}\right\rangle+\left\langle e^{2} \mid f^{1}\right\rangle .
$$

PROOF. The following coordinate transformation is introduced

$$
\underbrace{\left[\begin{array}{c}
\bar{t}_{\delta} \\
\bar{t}_{b} \\
\bar{t}_{b}^{0} \\
\bar{t}_{b}^{S}
\end{array}\right]}_{\bar{f}}=\left[\begin{array}{cccc}
\operatorname{Ad}_{b}^{n} & 0 & 0 & 0 \\
0 & \operatorname{Ad}_{b}^{n} & 0 & 0 \\
0 & 0 & \operatorname{Ad}_{b}^{n}(0) & 0 \\
0 & 0 & 0 & \operatorname{Ad}_{b}^{n}(S)
\end{array}\right]\left[\begin{array}{c}
t_{\delta} \\
t_{b} \\
t_{b}^{0} \\
t_{b}^{S}
\end{array}\right] .
$$

$$
\underbrace{\left[\begin{array}{c}
\bar{w}_{\delta} \\
\bar{w}_{b} \\
\bar{w}_{b}^{0} \\
\bar{w}_{b}^{S}
\end{array}\right]}_{\bar{e}}=\left[\begin{array}{cccc}
\operatorname{Ad}_{b}^{n} & 0 & 0 & 0 \\
0 & \operatorname{Ad}_{b}^{n} & 0 & 0 \\
0 & 0 & \operatorname{Ad}_{b}^{n}(0) & 0 \\
0 & 0 & 0 & \operatorname{Ad}_{b}^{n}(S)
\end{array}\right]^{-\mathrm{T}}\left[\begin{array}{c}
w_{\delta} \\
w_{b} \\
w_{b}^{0} \\
w_{b}^{S}
\end{array}\right] .
$$

It is clear that the coordinate transformation is power-conserving, that is

$$
\langle\bar{e} \mid \bar{f}\rangle=\langle e \mid f\rangle .
$$

Replacing $f, e$ with $\bar{f}, \bar{e}$, Equations (1), (2) and (3) become

$$
\begin{aligned}
\bar{t}_{\delta} & =\mathrm{d} \bar{t}_{b}, \\
\bar{w}_{b} & =\mathrm{d} \bar{w}_{\delta}, \\
\bar{t}_{b}(0) & =\bar{t}_{b}^{0}, \\
\bar{w}_{b}(0) & =\bar{w}_{b}^{0}, \\
\bar{t}_{b}(S) & =\bar{t}_{b}^{S}, \\
\bar{w}_{b}(S) & =\bar{w}_{b}^{S} .
\end{aligned}
$$

Let the subspace $\overline{\mathcal{D}}$ be defined by

$$
\begin{aligned}
\overline{\mathcal{D}}= & \left\{(\bar{f}, \bar{e}) \in \mathcal{F} \times \mathcal{E}: \bar{t}_{\delta}=\mathrm{d} \bar{t}_{b}, \bar{w}_{b}=\mathrm{d} \bar{w}_{\delta},\right. \\
& \bar{t}_{b}(0)=\bar{t}_{b}^{0}, \bar{w}_{b}(0)=\bar{w}_{b}^{0}, \\
& \left.\bar{t}_{b}(S)=\bar{t}_{b}^{S}, \bar{w}_{b}(S)=\bar{w}_{b}^{S}\right\} .
\end{aligned}
$$

The subspace $\overline{\mathcal{D}}$ is a Cartesian product of three Stokes-Dirac structures (see (van der Schaft and Maschke, 2002) for the definition of Stokes-Dirac structure) and thus it is a Dirac structure by virtue of example 5.1.5 in (Golo, 2002). Since the coordinate transformation is power-conserving then also the subspace $\mathcal{D}$ is a Dirac structure by virtue of Proposition 5.1.8 in (Golo, 2002).

Since $\mathcal{D}$ represents a Dirac structure then Equations (1), (2) and (3) describe the interconnection structure of the beam model.

\subsection{Energy-storage part}

The energy variables associated with the beam are the infitesimal deformation $q \in \Omega_{1}^{3}[0, S]$ and the infitesimal momenta $p \in \Omega_{1}^{3}[0, S]$. The infitesimal deformation is represented by

$$
q=\left[\begin{array}{l}
q_{\theta} \\
q_{x} \\
q_{y}
\end{array}\right],
$$

and the infitesimal momenta is represented by

$$
p=\left[\begin{array}{l}
p_{\theta} \\
p_{x} \\
p_{y}
\end{array}\right] .
$$

The infitesimal elastic energy denoted by $H_{q}(q)$ is given by

$$
H_{q}(q)=\frac{1}{2} * q^{\mathrm{T}} C^{-1} q,
$$

where $*$ is the Hodge operator defined with respect to the Euclidean metric along the line and $C \in$ $\mathbb{R}^{3 \times 3}$ is the matrix of compliance represented by

$$
C=\left[\begin{array}{ccc}
c_{\theta} & 0 & 0 \\
0 & c_{x} & 0 \\
0 & 0 & c_{y}
\end{array}\right] .
$$

which is in general also a function of $s$. The infitesimal kinetic energy denoted by $H_{p}(p)$ is given by

$$
H_{p}(p)=\frac{1}{2} * p^{\mathrm{T}} M^{-1} p
$$

where $M \in \mathbb{R}^{3 \times 3}$ is the mass matrix given by

$$
M=\left[\begin{array}{ccc}
\iota & 0 & 0 \\
0 & \mu & 0 \\
0 & 0 & \mu
\end{array}\right] .
$$

Here $\iota$ is the momentum of inertia per unit length and $\mu$ is the mass per unit-length. The total energy of the beam is then

$$
H=\int_{0}^{S}\left(\frac{1}{2} * p^{\mathrm{T}} M^{-1} p+\frac{1}{2} * q^{\mathrm{T}} C^{-1} q\right) .
$$




\subsection{Dynamical model}

The co-energy variables are the twist $t_{b}$ and the wrench $w_{\delta}$. These variables are related to the energy variables as

$$
\begin{gathered}
w_{\delta}=* C^{-1} q, \\
t_{b}=* M^{-1} p .
\end{gathered}
$$

The variables $t_{\delta}, w_{b}$ represent the rates of energy variables, that is

$$
\begin{aligned}
& \frac{\mathrm{d} q}{\mathrm{~d} t}=t_{\delta}, \\
& \frac{\mathrm{d} p}{\mathrm{~d} t}=w_{b}+p \wedge t_{b} .
\end{aligned}
$$

The linear transformation $p \wedge \Omega_{0}^{3} \rightarrow \Omega_{1}^{3}$ is defined as

$$
p \wedge=\left[\begin{array}{ccc}
0 & -p_{y} & p_{x} \\
p_{y} & 0 & 0 \\
-p_{x} & 0 & 0
\end{array}\right]
$$

The term $p \wedge t_{b}$ is the consequence of the description of the relative motion in "body" frame. Inserting the previous two relations into Equations (1), (2) and (3) gives the dynamical model of the beam, i.e.

$$
\begin{gathered}
{\left[\begin{array}{c}
\frac{\mathrm{d} q}{\mathrm{~d} t} \\
\frac{\mathrm{d} p}{\mathrm{~d} t}
\end{array}\right]=\left[\begin{array}{cc}
0 & \operatorname{Ad}_{n}^{b} \mathrm{dAd}_{b}^{n} \\
\left(\operatorname{Ad}_{b}^{n}\right)^{\mathrm{T}} \mathrm{d}\left(\mathrm{Ad}_{n}^{b}\right)^{\mathrm{T}} & p \wedge
\end{array}\right]\left[\begin{array}{c}
* C^{-1} q \\
* M^{-1} p
\end{array}\right]} \\
\left.C^{-1} q\right|_{s=0}=w_{b}^{0} \\
\left.C^{-1} q\right|_{s=S}=w_{b}^{S} \\
\left.M^{-1} p\right|_{s=0}=t_{b}^{0} \\
\left.M^{-1} p\right|_{s=S}=t_{b}^{S}
\end{gathered}
$$

The obtained dynamical model represents portHamiltonian system whose interconnection structure is described by Equations (1), (2) and (3) and with the Hamiltonian given by Equation (4).

\section{RELATIONS WITH OTHER BEAM MODELS}

In this section we compare the obtained model with the existing liner beam models. First relations for the infitesimal change of $x$ and $y$ along the beam are determined. Based on Figure 4 the following relation is obtained

$$
\begin{gathered}
x(s+a)-x(s)=\frac{a}{2}(\cos (\theta(s))+\cos (\theta(s+a)))+ \\
\cos (\theta(s+a)) q_{x}(s)-\sin \left(\theta(s+a) q_{y}(s) .\right.
\end{gathered}
$$

If $a$ tends to zero then the previous relation becomes

$$
\mathrm{d} x=* \cos (\theta)+\cos (\theta) q_{x}-\sin (\theta) q_{y} .
$$

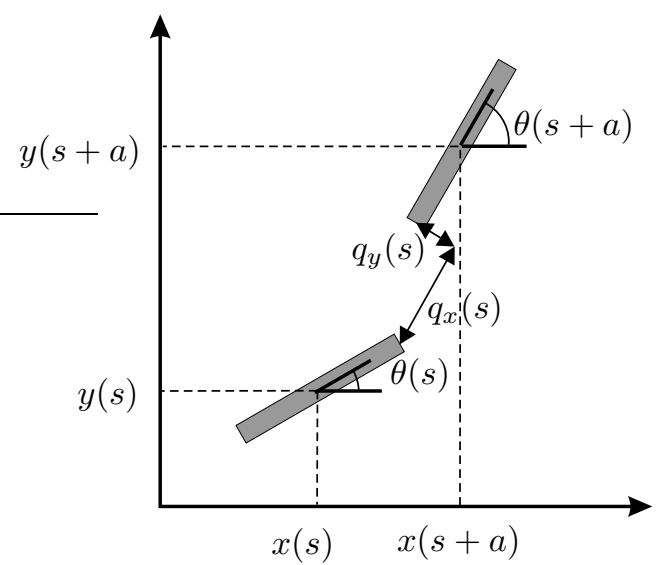

Fig. 4. Two finite elements.

The Hodge operator appears since all terms on both sides have to be one-forms. Similarly, the infitesimal change of $y$ has the following form

$$
\mathrm{d} y=* \sin (\theta)+\sin (\theta) q_{x}+\cos (\theta) q_{y} .
$$

Inserting the expressions for $\operatorname{Ad}_{b}^{n}$ and $\operatorname{Ad}_{n}^{b}$ into the term $\operatorname{Ad}_{n}^{b} \mathrm{dAd}_{b}^{n}$ and taking into account the previous two relations yields

$$
\operatorname{Ad}_{n}^{b} \operatorname{dAd}_{b}^{n}=\left[\begin{array}{ccc}
\mathrm{d} & 0 & 0 \\
q_{y} & \mathrm{~d} & -q_{\theta} \\
-*-q_{x} & q_{\theta} & \mathrm{d}
\end{array}\right],
$$

since $q_{\theta}=\mathrm{d} \theta$. Similarly $\left(\operatorname{Ad}_{b}^{n}\right)^{\mathrm{T}} \mathrm{d}\left(\mathrm{Ad}_{n}^{b}\right)^{\mathrm{T}}$ becomes

$$
\left(\operatorname{Ad}_{b}^{n}\right)^{\mathrm{T}} \mathrm{d}\left(\mathrm{Ad}_{n}^{b}\right)^{\mathrm{T}}=\left[\begin{array}{ccc}
\mathrm{d} & -q_{y} & *+q_{x} \\
0 & \mathrm{~d} & -q_{\theta} \\
0 & q_{\theta} & \mathrm{d}
\end{array}\right] .
$$

The term $P=\operatorname{Ad}_{n}^{b} \mathrm{dAd}_{b}^{n}$ can be split as

$$
P=\underbrace{\left[\begin{array}{ccc}
\mathrm{d} & 0 & 0 \\
0 & \mathrm{~d} & 0 \\
-* & 0 & \mathrm{~d}
\end{array}\right]}_{P_{\text {con }}}+\underbrace{\left[\begin{array}{ccc}
0 & 0 & 0 \\
q_{y} & 0 & -q_{\theta} \\
-q_{x} & q_{\theta} & 0
\end{array}\right]}_{P_{\text {mod }}} .
$$

The first part represents the constant part of $P$ and the second part represents the modulated part of $P$. Similarly the term $P^{*}=\left(\operatorname{Ad}_{b}^{n}\right)^{\mathrm{T}} \mathrm{d}\left(\operatorname{Ad}_{n}^{b}\right)^{\mathrm{T}}$ can be split as

$$
P^{*}=\underbrace{\left[\begin{array}{lll}
\mathrm{d} & 0 & * \\
0 & \mathrm{~d} & 0 \\
0 & 0 & \mathrm{~d}
\end{array}\right]}_{P_{\text {con }}^{*}}+\underbrace{\left[\begin{array}{ccc}
0 & -q_{y} & q_{x} \\
0 & 0 & -q_{\theta} \\
0 & q_{\theta} & 0
\end{array}\right]}_{P_{\bmod }^{*}} .
$$

\subsection{Wave equation and Timoshenko beam model}

If we are interested in dynamics of the beam with small deviations around the equilibrium corresponding to the unstressed conditions then the modulated terms $P_{\text {mode }}, P_{\text {mode }}^{*}$ and $p \wedge$ can be neglected. Consequently, Equation (5) splits into two parts. First of them given by

$$
\left[\begin{array}{c}
\frac{\mathrm{d} q_{x}}{\mathrm{~d} t} \\
\frac{\mathrm{d} p_{x}}{\mathrm{~d} t}
\end{array}\right]=\left[\begin{array}{ll}
0 & \mathrm{~d} \\
\mathrm{~d} & 0
\end{array}\right]\left[\begin{array}{c}
* c_{x} q_{x} \\
* \mu^{-1} p_{x}
\end{array}\right]
$$




$$
\begin{gathered}
\left.c_{x} q_{x}\right|_{s=0}=F_{x}^{0}, \\
\left.c_{x} q_{x}\right|_{s=S}=F_{x}^{S}, \\
\left.\mu^{-1} p_{x}\right|_{s=0}=v_{x}^{0}, \\
\left.\mu^{-1} p_{x}\right|_{s=S}=v_{x}^{S},
\end{gathered}
$$

describes the longitudinal motion of the beam and has the form of the rod equation. The second of them given by

$$
\begin{gathered}
{\left[\begin{array}{c}
\frac{\mathrm{d} q_{\theta}}{\mathrm{d} t} \\
\frac{\mathrm{d} q_{y}}{\mathrm{~d} t} \\
\frac{\mathrm{d} p_{\theta}}{\mathrm{d} t} \\
\frac{\mathrm{d} p_{y}}{\mathrm{~d} t}
\end{array}\right]=\left[\begin{array}{cccc}
0 & 0 & \mathrm{~d} & 0 \\
0 & 0 & -* & \mathrm{~d} \\
\mathrm{~d} & * & 0 & 0 \\
0 & \mathrm{~d} & 0 & 0
\end{array}\right]\left[\begin{array}{c}
* c_{\theta} q_{\theta} \\
* c_{y} q_{y} \\
* \iota^{-1} p_{\theta} \\
* \mu^{-1} p_{y}
\end{array}\right],} \\
\left.c_{\theta} q_{\theta}\right|_{s=0}=M^{0},\left.c_{\theta} q_{\theta}\right|_{s=S}=M^{S} \\
\left.c_{y} q_{y}\right|_{s=0}=F_{y}^{0},\left.c_{y} q_{y}\right|_{s=S}=F_{y}^{S} \\
\left.\iota^{-1} p_{\theta}\right|_{s=0}=\omega^{0},\left.\iota^{-1} p_{\theta}\right|_{s=S}=\omega^{S} \\
\left.\mu^{-1} p_{y}\right|_{s=0}=v_{y}^{0},\left.\mu^{-1} p_{y}\right|_{s=S}=v_{y}^{S} .
\end{gathered}
$$

describes the transversal motion of the beam and has the form of the Timoshenko beam model (see (Golo et al., 2002) for details).

\section{CONCLUSION}

In this paper, the dynamical model describing the beam with large deviations has been derived. It has been proved that the obtained model corresponds to a port-Hamiltonian system with modulated interconnection structure. If usual assumptions from linear beam theory apply then the obtained model can be split into two parts. The first part describes longitudinal motion (wave equations) and the second part represents transversal motion (Timoshenko beam model).

Extension of the obtained model for planar beams to three dimensional beams is straightforward. A three dimensional beam would be described by Equation (5) where $p$ and $q$ are six dimensional vectors, and $C, M, \operatorname{Ad}_{b}^{n}(s)$ are six dimensional matrices or even geometrically using Lie-algebra valued forms.

Future work will be focused on spatial discretization of the obtained model such that the energetic port-structure of the system is preserved. Also the proposed methodology for modeling beams will be applied to flexible shells and plates.

\section{REFERENCES}

Dalsmo, M. and A.J. van der Schaft (1999). On representation and integrability of mathematical structures in energy-conserving physical systems. SIAM Journal on Control and Optimization 37, 54-91.
Golo, G. (2002). Interconnection Structures in Port-Based Modelling: Tools for Analysis and Simulation. PhD thesis. University of Twente.

Golo, G., V. Talasila and A.J. van der Schaft (2002). A Hamiltonian formulation of the timoshenko beam model. In: Mechatronics 2002. Drebbel Institute for Mechatronics. Full paper on accompaning CD-rom.

Olver, P. (1993). Application of Lie groups to differential equations. Springer-Verlag.

Ortega, R., A.J. van der Schaft, I. Mareels and B.M. Maschke (2001). Putting energy back in control. Control Systems Magazine 21, 18-33.

Selig, J.M. and X. Ding (2001). A screw theory of static beams. In: International Conference on Intelligent Robots and Systems. Maui, Hawaii, USA, Oct. 29 - Nov. 03. pp. 312-317.

Stramigioli, S. (2001). Modeling and IPC Control of Interactive Mechanical Systems: A Coordinate-free Approach. Springer-Verlag.

van der Schaft, A.J. (2000). L $L_{2}$-Gain and Passivity Techniques in Nonlinear Control. Springer-Verlag.

van der Schaft, A.J. and B.M. Maschke (1995). The Hamiltonian formulation of energy conserving physical systems with external ports. Archiv fur Elektronik und Ubertragungstechnik 49, 362-371.

van der Schaft, A.J. and B.M. Maschke (2002). Hamiltonian formulation of distributed parameter systems with boundary energy flow. Journal of Geometry and Physics 42, 166194. 